\title{
Modeling Signal Strength of Body-Worn Devices
}

\author{
Alexander Kurusingal, Ashay Dhamdhere and Vijay Sivaraman \\ School of Electrical Engineering and Telecommunications, University of New South Wales, Sydney, NSW, 2052, Australia \\ Email: alexk@student.unsw.edu.au, ashay@unsw.edu.au,vijay@unsw.edu.au
}

\begin{abstract}
Body-wearable devices for physiological monitoring are fast becoming a reality - by 2014,420 million wearable wireless devices are expected to be in use, of which $90 \%$ will be for sports and fitness applications. We envisage the use of ultra-lightweight wearable devices for monitoring athletes in field sports such as soccer for quantifying, assessing and improving game performance. To this end, in this paper we present an empirical characterization of the radio signal strength of sensor devices mounted on an athlete's body. We fit simple analytical models to our empirical data, highlighting how the signal degrades with distance as well as orientation of the body. Our model aids in improved protocol design and locationing services that take into account propagation effects of the human body.
\end{abstract}

\section{INTRODUCTION}

With advances in device technology, ultra-light-weight, lowpower and low-cost wireless sensor devices are emerging that can be worn unobtrusively on the human body, and monitor physiological parameters on a continuous basis. Two applications of particular interest are home-based monitoring of patients with chronic medical conditions, and the monitoring of athletes during training and competition.

The miniaturization of sensor devices has led to two consequences of particular concern in the design of body area sensor networks. First, their body-mounted nature implies that the antenna is now located very close to the human body, drastically changing the propagation characteristics. Second, the small battery capacity and size leads to a significantly reduced transmit power compared to traditional sensor networks. These changes require a thorough reassessment of radio propagation models in the context of body area networks.

Much of the existing work on wireless networks makes simplifying assumptions on the radio propagation (such as circular radio ranges) when designing networks and analyzing performance. This might be a reasonable assumption in the presence of isotropic antennas mounted away from absorbing materials, but is unrealistic when the device is mounted directly on the human body. To the best of our knowledge very little work has been done on deriving these characteristics.

The contributions in this work are twofold. First, we experimentally profile the radio propagation characteristics of a body-mounted sensor device. We capture the variation in the received signal strength with both angular orientation and radial distance from the transmitter and show that this pattern differs significantly from an isotropic radiation pattern. Second, we derive an analytical model for the signal strength as a function of the orientation and distance from the transmitter. This makes available to the community an accurate characterization which can lead to more realistic performance evaluation of body-area sensor network protocols and signalstrength based locationing systems.

\section{RELATED WORK}

The performance of any sensor network relies inherently on the underlying radio propagation model. From a theoretical standpoint there has been much interest in finding the maximum throughput achievable in a wireless sensor network [1]. However, for the purposes of analytical tractability simplistic models for radio propagation have typically been assumed. These models assume that radio propagation is circular on a horizontal plane, with perfect reception inside the circular disk and no reception outside it. Such models have also been used to assess and compare the performance of routing protocols in wireless sensor networks [2].

Clearly, these models were meant to provide a high level estimate of certain network parameters and not for situations where accurate radio characterization is required. Furthermore, early applications of sensor networks typically involved isotropic antennas mounted away from absorbing surfaces. Increasingly, however, wireless devices have been getting closer and closer to the human body. For instance, [3] evaluates the effect of the human body on the propagation characteristics of different types of $\mathrm{Wi}-\mathrm{Fi}$ antennas found in portable computers. The authors show that there is a $25 \mathrm{~dB}$ loss in the signal emanating from the wireless device when the human body is in the way. However, while the device in this case is close to the human body, it is not mounted on it.

One of the earliest works on body-mounted devices explored the wireless channel characteristics between wireless devices mounted on the same body [4]. The authors used different antenna types to profile the channel between a transmitter mounted on the hip and multiple receivers on the upper body. It was found that the absorption due to the water content of the human body resulted in a $40 \mathrm{~dB}$ path loss between the transmitter and receiver. While significant, the transmitter and receiver in this case were both mounted on the human body. On the other hand, we are interested in body-mounted devices communicating with remote base stations, as would be deployed in athlete monitoring applications.

Signal propagation models are also essential for performing signal strength based locationing. Prior work in this area [5] has focused on determining the location of objects in an indoor setting such as a warehouse, and takes into account only the distance (and not the orientation) of the inanimate object. As 
we shall show, when the object in question is a human being, both distance and orientation are important.

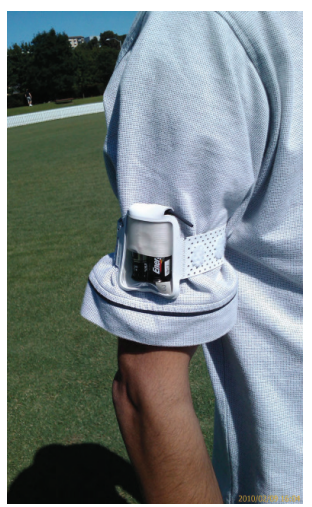

Fig. 1. MicaZ mote mounted on arm

From our experiments with first division soccer players, we were able to show that link correlations exist between soccer players due to their co-ordinated motion [6]. We have also created a multi-hop protocol which balances resource consumption and delay to deliver data in an optimal manner [7].

\section{EXPERIMENTAL PROFILING}

Our experimental work profiles how the human body influences radio propagation between the transmitter and receiver. We utilized micaZ motes from Crossbow Technologies [8] running the TinyOS operating system as our transmitter and receiver. As the transmitter represented the body-worn sensor device, it used a $1 / 4$ wavelength dipole antenna that comes standard with the Mica motes. The receiver represented a base station (perhaps located on the periphery of the soccer field in an athlete monitoring application), and therefore used a bigger high-gain (+12dBi) antenna from TP-Link [9].

Our objective was to characterize how the received signal strength varied with the relative position between the transmitter and receiver. The transmitter sent packets at a fixed rate of 4 packets per second, at a fixed power level of $1 \mathrm{~mW}$. Upon successfully receiving a packet, the receiver computed the Received Signal Strength Indicator (RSSI) of the received packet and sent this value to a laptop computer over the serial port. In the micaZ motes the RSSI value is an 8-bit number obtained by sampling the onboard ADC during packet reception. The RSSI value was then converted to a $\mathrm{dBm}$ value by subtracting 45 [10]. Our experiments were performed in an open soccer field away from any sources of interference.

With this intent we performed two experiments. Our objective in the first experiment was to ascertain the propagation pattern in free space, so as to have a baseline against which to compare the effect of the human body. The transmitter was mounted at the top of a non-conducting pole at a height of $1.5 \mathrm{~m}$ above ground level, while the receiver remained stationary. We then increased the distance between transmitter and receiver in steps of $1 \mathrm{~m}$, and recorded the RSSI reading at each step. This was repeated until reliable reception could

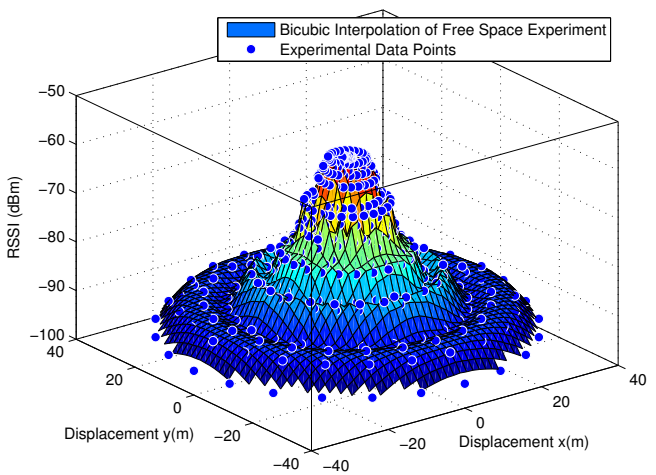

Fig. 2. Interpolated surface fit of Free Space Experimental Data

not be obtained (where reception was considered reliable when there was no packet loss.) As we expected, the RSSI showed no effect of the orientation between the transmitter and receiver, and depended only on the distance. The surface plot of the raw data from this experiment is plotted in Fig. 2.

Having set the baseline we were now in a position to evaluate the effect of the body. The second experiment proceeded similarly to the first, albeit with a crucial difference: the transmitter was mounted at a height of $1.5 \mathrm{~m}$ on the right arm of a test subject, as shown in Fig. 1. The subject rotated his body in 15 degree increments (from $0^{\circ} \rightarrow 345^{\circ}$ ) with respect to the receiver. The height of the test subject was $1.88 \mathrm{~m}$ tall, and the weight was $75 \mathrm{Kg}$; this corresponds to a BMI of 21 . The raw data from the second experiment is plotted in Fig. 3.

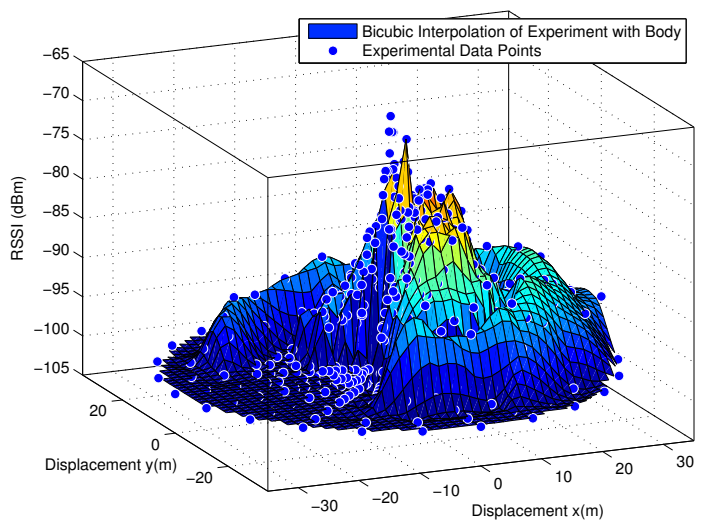

Fig. 3. Interpolated surface fit of Human Body from Experimental data

As expected, the RSSI contours now show very significant reduction in signal strength when the body is in the way. When the body is not blocking the signal, we observe $30 \mathrm{~m}$ of uninterrupted range. On the other side, however, the range is only $2 \mathrm{~m}$ before reception drops completely. The highest recorded RSSI occurred at the location $(0.707,0.707)$, corresponding to an orientation of $45^{\circ}$ and a range of $1 \mathrm{~m}$. 


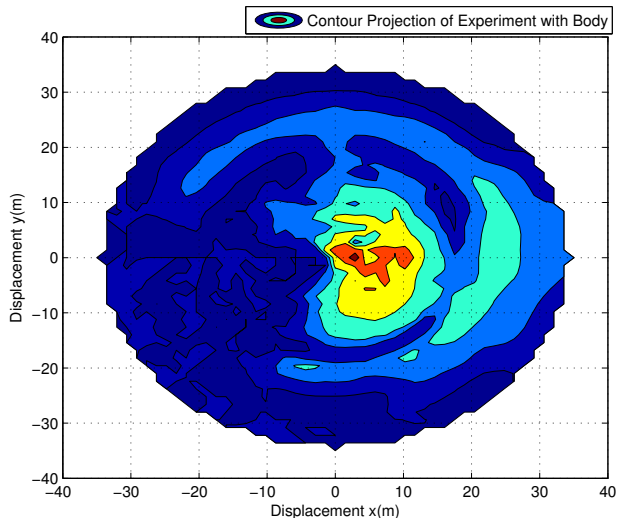

Fig. 4. Contour projection of Figure 3

\section{Analytical Formulation}

Our objective was to use the raw data to derive an analytical expression which captured the variation of signal strength, as a function of distance and orientation. In free space, the received signal strength typically falls with distance $r$ as a power-law:

$$
R S S I=a * r^{-b}
$$

where $b$ is close to 2.5 and $a$ is a proportionality constant incorporating effects of the antenna, transmit power and environmental variables. We chose to keep our formulation consistent with this notation, where the $a$ and $b$ terms now incorporate orientation. (a) Polar plot of $a_{\theta}$

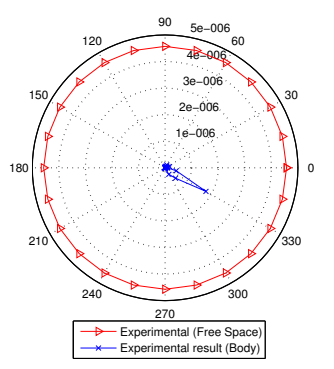

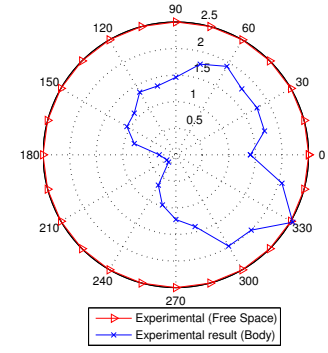

(b) Polar plot of $b_{\theta}$
Fig. 5. Polar plot of $a$ and $b$ terms in free space and on-body

For the case when the device is body-worn, we keep the same form for the received signal strength as in (1), but make parameters $a$ and $b$ depend on the angle $\theta$ at which the body is orientated relative to the receiver. The best-fit values of $a_{\theta}$ and $b_{\theta}$ for the various values of $\theta$ (at $15^{\circ}$ increments) obtained from the measured data are shown as polar plots in Fig. 5(a) and 5 (b) respectively.

We now try to model the dependence of $a_{\theta}$ and $b_{\theta}$ on $\theta$ analytically. For which we attempted to fit three different curve types: Fourier, Sum of Sines, and Gaussian. A visual representation of this fitting is shown in Fig. 6(a) for $a$ and in Fig. 6(b) for $b$. We evaluated each fitting method on the basis of simplicity of the expression and goodness of the fit.

For parameter $a$ it was found that fitting $\log _{10} a$ resulted in a better goodness of fit compared with fitting $a$, for the

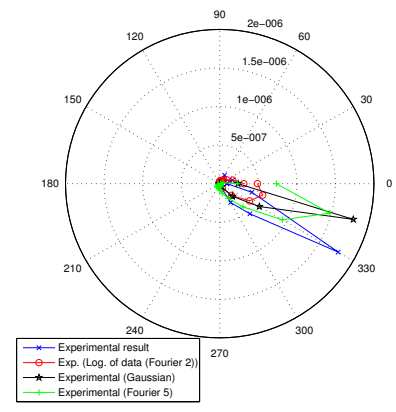

(a) $a_{\theta}$ and associated fits

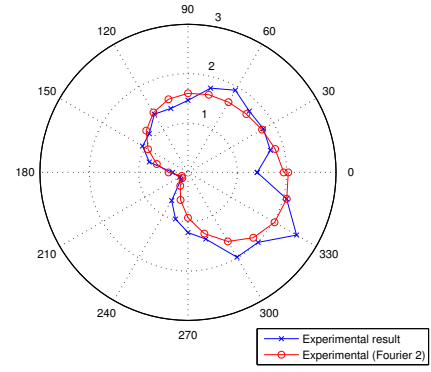

(b) $b_{\theta}$ and associated fits
Fig. 6. Polar plots of $a$ and $b$ terms and associated fits

same complexity of the expression. Further, it was found that $\log _{10} a$ was best represented by a second order Fourier series as shown in eq. (2), with an $R^{2}$ value of 0.9603 . A better fit is possible with a Gaussian curve (with an $R^{2}$ value of 0.9622 ), however the expression is far more complicated.

$$
a=10^{-f_{a}}
$$

$$
\begin{array}{r}
f_{a}=7.868-1.551 * \cos (\theta * 0.9893) \\
-0.1774 * \sin (\theta * 0.9893)+0.1882 * \cos (2 * \theta * 0.9893) \\
+0.5404 * \sin (2 * \theta * 0.9893)
\end{array}
$$

Likewise, $b$ was fitted best by a second order Fourier series as shown in (4), with an $R^{2}$ value of 0.9033 .

$$
\begin{array}{r}
b=1.27+0.8086 * \cos (\theta * 0.9726)+ \\
0.1851 * \sin (\theta * 0.9726)-0.1396 * \cos (2 * \theta * 0.9726) \\
-0.3049 * \sin (2 * \theta * 0.9726)
\end{array}
$$

It has been shown that the human body absorbs radio signals at $2.4 \mathrm{GHz}$. As a result, we expect very high attenuation for orientations where the body significantly shadows the signal (it has been further shown that at these orientations the dominant component of the signal arises from creeping waves around the body [4] ). It should be noted that we are not measuring the signal directly on the body, however, the above results are still significant especially at orientations between 150 and 270 degrees.

Noting further that the $a$ values denote the received signals at a distance of $1 \mathrm{~m}$ from the transmitter, along a given orientation, we see that $a_{180}\left(=10^{-9.646}\right)$ is two orders of magnitude below $a_{0}\left(=10^{-7.028}\right)$; this loss is consistent with the loss experienced by a creeping wave traveling halfway around the human body. Similarly, we compare the $b$ values at different orientations. It is found that at orientations between 90 and 270 degrees (where the wireless range is significant), the $b$ values lie in the range 1.2 to 2 . While this is a higher value than that for free space (leading to a slower decay), it is combined with a much lower value of $a$, as compared to free space. 


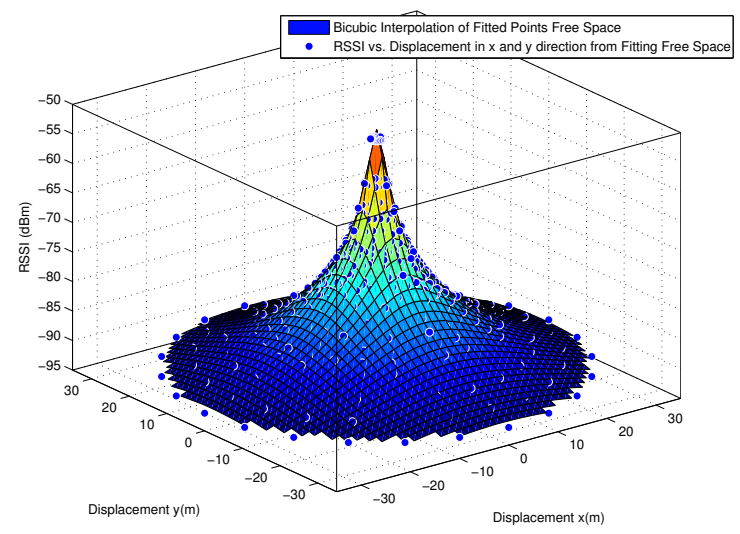

Fig. 7. Interpolated surface fit generated by model for Free Space

Given these equations, the following figures were created. The data points for these figures were obtained by putting the values from (2) and (4) into (1). Firstly, Fig. 7 shows the interpolated surface obtained using the fitted Free Space parameters. It comes as no surprise that the interpolated surface becomes conical in shape due to (1).

Fig. 8 shows a 3D surface which was interpolated using data points generated by substituting Eq. (2) and (4) into (1). The dots shown in Fig. 8 are the experimental data points obtained from Experiment 2. The contour projection of this surface is given in Fig. 9. Comparing Fig. 4 and 9, we see that the model predicts close to $40 \mathrm{~m}$ of uninterrupted coverage in the right hand direction, whereas the experimental result suggests that a little over $30 \mathrm{~m}$ range is possible.

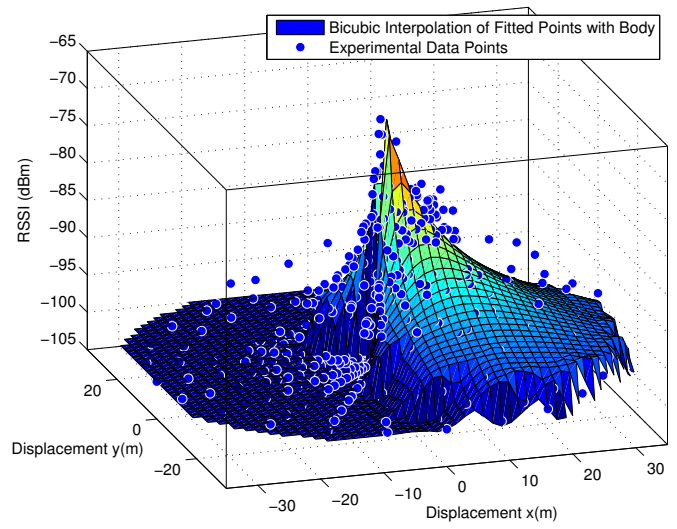

Fig. 8. Interpolated surface fit generated by model for Human Body

\section{CONClusion}

In the existing research, only [5] has modeled location as a function of distance from the transmitter, but they do not take into account the effect of the human body. [4] and [3] do show how $2.4 \mathrm{GHz}$ radio signals are affected by the human body, but they do not use it in the context of locationing or sensor networks. In this work we profile for the first time the radio propagation characteristics of low power body-worn

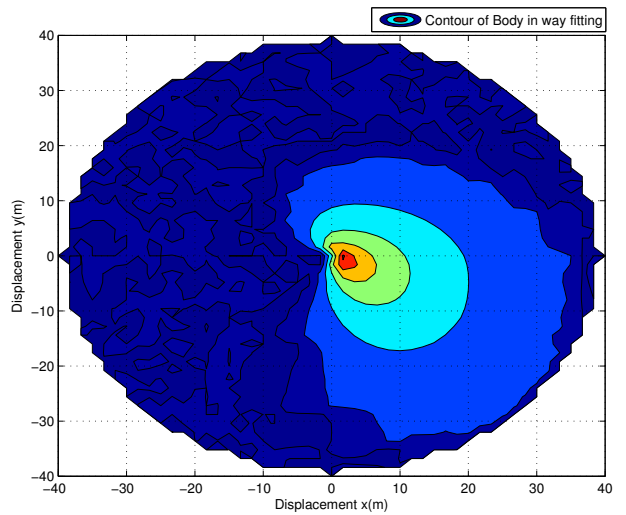

Fig. 9. Contour projection of Figure 8

sensor devices which communicate with fixed, non body-worn base stations.

We first show that in the absence of the human body the propagation patterns follow the classical inverse square law model. Next we show that the propagation patterns change drastically when the transmitter is mounted on the human body, with the received signal strength exhibiting strong directionality. Finally, we formulate an analytical expression for the RSSI at any distance and orientation from the transmitter, enabling not only a more accurate characterization of body area network performance, but also specialized services such as RSSI based locationing.

In future we plan to repeat the experiments with more test subjects. This should show how the contours and the resulting functions will vary across different body types.

\section{REFERENCES}

[1] P. Gupta and P. Kumar, "The capacity of wireless networks," Information Theory, IEEE Transactions on, vol. 46, no. 2, pp. 388-404, Mar 2000.

[2] J. Al-Karaki and A. Kamal, "Routing Techniques in Wireless Sensor Networks: A Survey," IEEE Wireless Communications, vol. 11, no. 6, pp. 6-28, Dec. 2004

[3] J. Guterman, A. Moreira, C. Peixeiro, and Y. Rahmat-Samii, "Comparison study of electromagnetic human interaction with various $2.4 \mathrm{ghz}$ laptop integrated antennas," May 2008, pp. 1-4.

[4] Y. Hao, A. Alomainy, P. Hall, Y. Nechayev, C. Parini, and C. Constantinou, "Antennas and propagation for body centric wireless communications," in IEEE/ACES International Conference on Wireless Communications and Applied Computational Electromagnetics, April 2005, pp. $586-589$.

[5] S. Hara, D. Zhao, K. Yanagihara, J. Taketsugu, K. Fukui, S. Fukunaga, and K. Kitayama, "Propagation Characteristics of IEEE 802.15.4 Radio Signal and Their Application for Location Estimation," in 61st IEEE Vehicular Technology Conference, 2005, vol. 1, May 2005, pp. 97 101 Vol. 1.

[6] V. Sivaraman, S. Grover, A. Kurusingal, A. Dhamdhere, and A. Burdett, "Experimental study of mobility in the soccer field with application to Real-Time athlete monitoring," in IEEE International Conference on Wireless and Mobile Computing, Networking and Communications (WiMob'2010), 2010.

[7] A. Dhamdhere, H. Chen, A. Kurusingal, V. Sivaraman, and A. Burdett, "Experiments with wireless sensor networks for Real-Time athlete monitoring," in 5th IEEE International Workshop on Practical Issues in Building Sensor Network Applications (SenseApp 2010), 2010.

[8] "Crossbow Technology - Mica2 and MicaZ motes." [Online]. Available: http://www.xbow.com

[9] “TP-Link TL-ANT2412D Antenna." [Online]. Available: http://www. tp-link.com/products/print.asp?pmodel=TL-ANT2412D

[10] "TI CC2420 Radio transciever." [Online]. Available: http://focus.ti.com/ lit/ds/symlink/cc2420.pdf 